\title{
Intravenous Diuretic, Inotropic or Vasodilator Augmentation for Heart
}

\section{Failure}

National Cancer Institute

\section{Source}

National Cancer Institute. Intravenous Diuretic, Inotropic or Vasodilator Augmentation

for Heart Failure. NCI Thesaurus. Code C119208.

Initiation or intensification of intravenous diuretics, inotropics or vasodilators to treat heart failure. 